\section{Research Square}

Preprints are preliminary reports that have not undergone peer review.

They should not be considered conclusive, used to inform clinical practice, or referenced by the media as validated information.

\title{
Protective lleostomy Increased the Incidence of Rectal Stenosis After Anterior Resection of Rectal Cancer
}

Hui Zhang

Fudan University Shanghai Cancer Center https://orcid.org/0000-0001-8803-2696

Shanshan Li

Zoucheng People's Hospital

Xin Jin

Fudan University Shanghai Cancer Center

\section{Xian Wu}

Fudan University Shanghai Cancer Center

\section{Zhiyuan Zhang}

Fudan University Shanghai Cancer Center

\section{Lijun Shen}

Fudan University Shanghai Cancer Center

\section{Juefeng Wan}

Fudan University Shanghai Cancer Center

\section{Yan Wang}

Fudan University Shanghai Cancer Center

Yaqi Wang

Fudan University Shanghai Cancer Center

Wang Yang

Fudan University Shanghai Cancer Center

Menglong Zhou

Fudan University Shanghai Cancer Center

Jing Zhang

Fudan University Shanghai Cancer Center

Tao Lv

Fudan University Shanghai Cancer Center

Yun Deng

Fudan University Shanghai Cancer Center

\section{Fan Xia}

Fudan University Shanghai Cancer Center

Zhen Zhang ( $\nabla$ zhen_zhang@fudan.edu.cn ) 
Fudan University Shanghai Cancer Center https://orcid.org/0000-0001-7825-2679

\section{Research Article}

Keywords: rectal cancer, stenosis, preventive ileostomy, radiotherapy

Posted Date: January 19th, 2022

DOI: https://doi.org/10.21203/rs.3.rs-1258537/v1

License: (9) This work is licensed under a Creative Commons Attribution 4.0 International License. Read Full License 


\section{Abstract}

Background. In most of the views, rectal stenosis results from pelvic radiotherapy. However, patients without receiving radiotherapy also suffer stenosis. In this study, we evaluated the factors associated with rectal stenosis after anterior rectal resection (ARR).

Methods. We conducted a retrospective study with ARR patients who underwent neoadjuvant chemoradiotherapy and the patients without radiotherapy. Patients received watch and wait strategy with a clinical complete response after chemoradiotherapy were also included. Patients with colonoscopy follow-up were included for further analyses; 439 patients who underwent neoadjuvant chemoradiotherapy; 545 patients who received ARR without radiotherapy and 33 patients who received watch and wait strategy. Stenosis was defined as cannot pass a 12-mm colonoscopy. Univariate and multivariate logistic regression analyses were performed to identify variables associated with rectal stenosis.

Results. According to the multivariate analysis in patients receiving ARR, both protective stoma and preoperative radiotherapy affected the occurrence of stenosis, with the odds ratios (ORs) of 3.375 and 2.251, respectively. According to the multivariate analysis, a preventive ileostomy was the only factor associated with stenosis both in patients receiving preoperative radiotherapy and without radiotherapy. Non-reversal ileostomy and long time between ileostomy and restoration increased the possibility of stenosis. In 33 patients who received watch and wait strategy, only one patient (3\%) experienced stenosis.

Conclusion. Both surgery and radiotherapy are risk factors for rectal stenosis in rectal cancer patients. Compared to preoperative radiotherapy, a protective ileostomy is a more critical factor associated with rectal stenosis.

\section{Background}

Colorectal cancer (CRC) is the third most common malignancy among men and women [1], and rectal cancer accounts for up to $50 \%$ of all colorectal cancers. Neoadjuvant chemoradiotherapy followed by total mesorectal excision (TME) has become the standard care for T3-4 and node-positive tumors in rectal cancer [2]. However, after TME surgery, a certain number of patients will experience anastomotic complications, including anastomotic leakage and stenosis. Anastomotic leakage is a feared complication after anterior resection as it increases postoperative mortality. A temporary ileostomy created at the time of surgery may reduce the potential leak [3]. Rectal stenosis represents a challenging complication after rectal resection and can become a long-term complication among rectal cancer patients, it will seriously affect the quality of life in high-grade stenosis patients. The pathophysiology and contributing factors have only been partially understood. Postoperative anastomotic leakage and radiotherapy have been reported as predisposing factors [4-6]. In most of the views, pelvic radiotherapy is the most important reason for rectal stenosis. However, patients without receiving radiotherapy also suffer stenosis. Few studies published to date have analyzed the incidence of rectal stenosis after ARR in 
rectal cancer patients. This study evaluated the incidence and risk factors of rectal stenosis so that effective measurements can be adopted to mitigate the event.

\section{Patients And Methods}

\section{Patients and treatment}

All of the consecutive patients diagnosed with rectal adenocarcinoma and received anterior rectal resection (ARR) at Fudan University Shanghai Cancer Center between January 2006 and December 2018 were retrospectively studied, including receiving neoadjuvant chemoradiotherapy and without receiving radiotherapy. The exclusion criteria were short-course radiotherapy (5 Gy 5 fractions), no colonoscopy follow-up, abdominoperineal resection (APR), and palliative resection. Neoadjuvant chemoradiotherapy was indicated for patients with lesions of the lower and middle rectum as T3 or T4 and for those lymph nodes suspected of being metastatic. The intensity-modulated radiation therapy (IMRT) technique was performed with a photon beam of 6-MV energy. The planned treatment dose of radiation was $50 \mathrm{~Gy}$ in 25 fractions ( $2 \mathrm{~Gy} /$ fraction), 5 fractions/week. The RTOG contouring atlas was referenced for clinical tumor volume (CTV) contouring [7]. Concurrent chemotherapy was conducted in nearly all the patients, and concurrent chemotherapy regimens are listed in Table S1. Capecitabine was administered concurrently with radiotherapy on radiation days. Patients received capecitabine $825 \mathrm{mg} / \mathrm{m}^{2}$ bid orally on radiation days for the capecitabine regimen. Approximately ten years ago, we conducted a phase 2 clinical trial that explored oxaliplatin's role with capecitabine concurrent with radiotherapy in advanced rectal cancer patients (unpublished data). For the oxaliplatin regimen, patients received oxaliplatin $50 \mathrm{mg} / \mathrm{m}^{2}$ every week, concurrent with capecitabine $625 \mathrm{mg} / \mathrm{m}^{2}$ bid orally on radiation days. Recently, irinotecan and capecitabine concurrently with radiotherapy showed excellent effects in our phase 1, $2[8,9]$ and phase 3 trials [10]. Patients received capecitabine $\left(625 \mathrm{mg} / \mathrm{m}^{2}\right.$, bid) orally along with weekly irinotecan for 5 weeks according to the UGT1A1/28 genotype. The weekly irinotecan dose was $80 \mathrm{mg} / \mathrm{m}^{2}$ in patients with the $* 1 * 1$ genotype and $65 \mathrm{mg} / \mathrm{m}^{2}$ in those with the $* 1 * 28$ genotype. Surgery was undertaken following the principles of TME for patients. 439 patients were received neoadjuvant chemoradiotherapy following ARR and 545 patients underwent ARR without preoperative or postoperative radiotherapy.

We also retrospectively studied 33 patients who received non-operative strategy after achieved clinical complete response (cCR) between 2015 and 2020.

The potential risk factors analyzed for the stenosis were gender, age, tumor distance from the anal margin ( $\leq 5 \mathrm{~cm}$ or $>5 \mathrm{~cm}$ ), tumor staging ( $\mathrm{cT}$ and $\mathrm{cN}$ status were assessed by magnetic resonance imaging (MRI)), body mass index (BMI), smoking, drinking, hypertension, diabetes, radiotherapy dose (RT dose), concurrent chemotherapy, pattern of surgery (open or laparoscopic), occurrence of anastomotic fistula, protective ileostomy, tumor regression grade (TRG) score, and RT response (pCR[pathologic complete response]/almost pCR or poor response). The work was in accordance with The Code of Ethics 
of the World Medical Association (Declaration of Helsinsi) and was approved by the hospital's Medical Ethics Committee.

\section{Evaluation of rectal stenosis}

Rectal stenosis is difficult to diagnose and grade, colonoscopy is commonly used to evaluate stenosis. Patients were encouraged to engage in a regular follow-up after the operation. Colonoscopy was performed before the restoration of protective ileostomy or the first year after surgery, and every 2-3 years after that, or in case of new symptoms or suspected relapse. Anastomotic or rectal stenosis was defined as cannot pass a 12-mm colonoscopy. We found that stenosis was mainly located above the anastomoses, and not at the site of anastomoses. Colonoscopy was performed on 439 patients, who underwent neoadjuvant chemoradiotherapy followed by ARR. A group of 545 patients who received ARR without radiotherapy and underwent colonoscopy examination was formed to explore whether other factors affect rectal stenosis regardless of radiotherapy. Further, 33 patients who received nonoperative management when exhibiting cCR after neo-adjuvant chemoradiotherapy was analyzed for stenosis.

\section{Statistical analysis}

Statistical analysis was conducted using SPSS software. We analyzed the categorical variables using the chi-square test and the quantitative ones with the Student's $t$-test (mean and standard deviation [SD]). Initially, we performed a univariate analysis for each independent variable. Then, the candidates who had a $p$-value $\leq 0.05$ were considered for the multivariate model. $P<0.05$ was considered statistically significant and marked with asterisks (*).

\section{Results}

\section{Both protective ileostomy and preoperative radiotherapy were related to stenosis in patients receiving anterior rectal resection}

Combining the data of preoperative radiotherapy and non-radiotherapy, there were 155 patients presenting stenosis. Gender, tumor location, smoking, surgery pattern, protective ileostomy, and radiotherapy were significantly different between the stenosis and non-stenosis groups $\left(P<0.05^{*}\right.$, Table S2). After performing multivariate analysis, only protective ileostomy and radiotherapy were the significant factors (Table 1). In the stenosis group, 73.5\% of patients conducted protective ileostomy and $33.7 \%$ in the non-stenosis group. Protective ileostomy increased 3.375 the risk of stenosis. In patients with stenosis, $72.8 \%$ received preoperative radiotherapy, and the proportion was $38.4 \%$ in the non-stenosis group. Preoperative radiotherapy increased 2.251 the risk of stenosis. In 393 patients with an ileostomy, there were $29.0 \%$ (114/393) patients with stenosis and 6.9\% (41/591) patients suffering stenosis without ileostomy (Figure 1). In 424 patients with preoperative radiotherapy, 25.9\% (114/439) experienced stenosis, in contrast with $7.5 \%(41 / 545)$ in non-radiotherapy patients. 
Table 1

Multivariate logistic regression analysis of the association between the factors and stenosis in all non-radiotherapy and preoperative radiotherapy patients

\begin{tabular}{|c|c|c|c|c|c|}
\hline Variables & $\begin{array}{l}\text { Stenosis, } \\
\mathrm{N}=155\end{array}$ & No stenosis, $\mathrm{N}=829$ & OR & $95 \% \mathrm{Cl}$ & $P$ value \\
\hline Sex, n (\%) & & & 1.133 & $0.738-1.742$ & 0.568 \\
\hline Male & $108(69.7)$ & $493(59.5)$ & & & \\
\hline Female & $47(30.3)$ & $336(40.5)$ & & & \\
\hline Tumor location, n (\%) & & & 0.900 & $0.600-1.351$ & 0.611 \\
\hline$\leq 5 \mathrm{~cm}$ to anus & $58(37.4)$ & $182(22.0)$ & & & \\
\hline$>5 \mathrm{~cm}$ to anus & $97(62.6)$ & $647(78.0)$ & & & \\
\hline Smoking, n (\%) & & & 1.572 & $0.996-2.480$ & 0.052 \\
\hline Yes & $45(29.0)$ & $158(19.1)$ & & & \\
\hline No & $110(71.0)$ & $671(80.9)$ & & & \\
\hline Surgery, n (\%) & & & 1.039 & $0.689-1.568$ & 0.864 \\
\hline Open & $92(59.4)$ & $620(74.8)$ & & & \\
\hline Laparoscopic & $63(40.6)$ & $209(25.2)$ & & & \\
\hline Stoma, n (\%) & & & 3.375 & $2.083-5.470$ & $0.000^{*}$ \\
\hline Yes & $114(73.5)$ & $279(33.7)$ & & & \\
\hline No & $41(26.5)$ & $550(66.3)$ & & & \\
\hline Radiotherapy, n (\%) & & & 2.251 & $1.440-3.519$ & $0.000 *$ \\
\hline Yes & $110(72.8)$ & $314(38.4)$ & & & \\
\hline No & $41(27.2)$ & $504(61.6)$ & & & \\
\hline
\end{tabular}

\section{Protective ileostomy was the only independent factor related to stenosis in patients with preoperative chemoradiotherapy}


We identified 439 patients treated with neoadjuvant chemoradiotherapy followed by ARR and with followup data on colonoscopy. All the patients received intensity-modulated RT (IMRT) and TME surgery, and the total planned dose of 50 Gy in 25 fractions.

In patients with preoperative radiotherapy (Table S2), there are 25.9\% (114/439) patients experienced stenosis; the mean ages were 55.0 years (SD: \pm 10.5 ) and 54.7 years (SD: \pm 10.7 ) in the stenosis and nonstenosis group, respectively $(p>0.05)$. By performing a chi-square test (Table $\mathrm{S} 2)$, comorbidities, such as diabetes and hypertension, were not different between the stenosis and non-stenosis groups. The tumor location, BMI, history of drinking and smoking, cT stage, cN stage, RT dose, concurrent chemotherapy, the pattern of surgery, the occurrence of leakage, and TRG score were not significantly different between the two groups. Males, smoking and patients who underwent preventive ileostomy were more likely to suffer stenosis. In 114 patients with stenosis, 82 (71.9\%) were male, and 32 (28.1\%) were female; the proportion of males was much higher in the stenosis group $(P=0.047 *)$. More males $(29.1 \% ; 82 / 282)$ suffered from stenosis than females $(20.4 \% ; 32 / 157)$. More patients $(29.8 \%)$ had a history of smoking in the stenosis group than in the non-stenosis group $\left(19.4 \% ; P=0.026^{*}\right)$. A protective ileostomy was the most significantly different factor; $80.7 \%$ of patients received ileostomy in the stenosis group, while only $64.6 \%$ of patients had an ileostomy in the non-stenosis group $\left(P=0.001^{*}\right)$. After performing multivariate analysis (Table 2), the only statistically significant predictor of stenosis was the presence of ileostomy $\left(P=0.003^{\star}\right)$, and ileostomy increased the risk of stenosis by 2.2. Among the 302 patients receiving ileostomy, 92 $(30.5 \%)$ patients experienced stenosis, which is much higher than the $22(16.1 \%)$ patients with stenosis without ileostomy. 
Table 2

Multivariate logistic regression analysis of the association between the factors and stenosis in preoperative radiotherapy

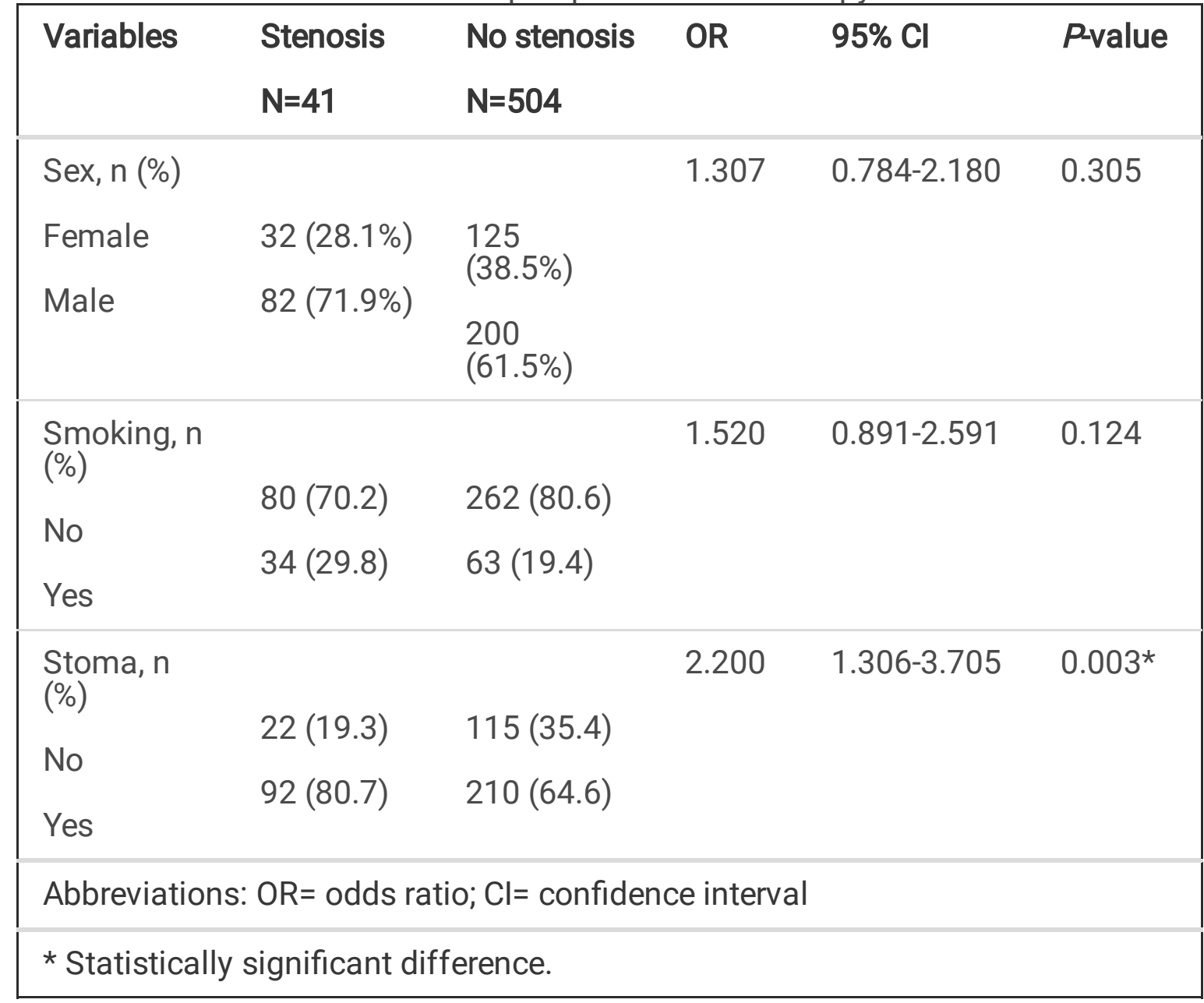

Of the 302 patients who had a protective ileostomy, 259 (85.8\%) patients had the ileostomy closed, and $53(17.5 \%)$ remained with the stoma. Only $65.2 \%$ patients received a restoration in the stenosis group, and $90 \%$ in the non-stenosis group $\left(P=0.001^{*}\right)$. Fifty-three patients did not receive restoration, 17 patients because of stenosis, 13 patients because of metastasis, 3 patients because of the presence of both stenosis and metastasis, 5 for leakage, 2 for relapse, and 14 for unknown reasons. To study if the interval between radiotherapy and ARR affected stenosis, we also analyzed the time from the end of radiotherapy to ARR. The result did not show a significant difference; the mean time in the stenosis and non-stenosis groups was $63.359 \pm 17.035$ days and $60.667 \pm 23.102$ days, respectively $(P=0.316)$. Furthermore, we calculated the interval between ARR and restoration, and anylzed the cut-off value using ROC(the receiving operator characteristic) curve, it showed that the cut-off value was about six months (Figure 2). Then, we divided the patients into those with less than six months from stomy to restoration and those that did not receive restoration within six months. Patients who did not receive restoration were defined as not receive restoration within six months. In the stenosis group, $81.5 \%$ of patients received restoration more than 6 months after ileostomy, and this proportion was only $52.4 \%$ in the non-stenosis group (Table 3). 
Table 3

The parameters of stoma and stenosis in patients received stoma

\begin{tabular}{|llll|}
\hline & $\begin{array}{l}\text { Stenosis } \\
\mathrm{N}=92\end{array}$ & $\begin{array}{l}\text { No stenosis } \\
\text { N }=210\end{array}$ & P-value \\
\hline Restored, $\mathrm{n}(\%)$ & & & $0.001^{*}$ \\
No & $32(34.8)$ & $21(10.0)$ & \\
Yes & $60(65.2)$ & $189(90.0)$ & \\
\hline Time from end of RT to surgery (d) & & & 0.316 \\
Mean \pm SD & $63.359 \pm 17.035$ & $60.667 \pm 23.102$ & \\
\hline Time from stoma to restoration, $\mathrm{n}(\%)$ & & & $0.001^{*}$ \\
$\leq 6 \mathrm{~m}$ & $17(18.5)$ & $100(47.6)$ & \\
$>$ 6m & $75(81.5)$ & $110(52.4)$ & \\
\hline * Statistically significant difference. & & & \\
\hline
\end{tabular}

\section{Protective ileostomy was the only factor related to stenosis in patients without radiotherapy}

In most of the views, stenosis is the late side effect of radiotherapy. To explain this problem, we also studied if patients without radiotherapy will present with stenosis. Five hundred forty-five patients who did not receive radiotherapy but had colonoscopy follow-up was included. The incidence rate of stenosis was 7.5\% (41/545). By performing a chi-square test (Table S3), the clinical characteristics were not significantly different between the stenosis and non-stenosis groups for gender, smoking, drinking, hypertension, diabetes, pT stage, pN stage, and the occurrence of leakage. However, age, tumor location, surgery pattern and protective ileostomy were imbalanced between the two groups. Older age, tumor in the lower location, and laparoscopic surgery increased the incidence of stenosis. After performing the multivariate analysis (Table 4), only protective ileostomy was statistically significant for stenosis $(P=0.001 *)$. In the stenosis group, $80.7 \%$ of the patients received protective ileostomy, while only $64.6 \%$ in the non-stenosis group did. The odd ratio of stomy was 2.533 , and a similar result was obtained for the preoperative radiotherapy patients. 
Table 4

Multivariate logistic regression analysis of the association between the factors and stenosis in non-preoperative radiotherapy

\begin{tabular}{|c|c|c|c|c|c|}
\hline Variables & Stenosis & No stenosis & OR & $95 \% \mathrm{Cl}$ & $P$ value \\
\hline Age (y) & $61.5 \pm 10.8$ & $57.8 \pm 10$ & 0.999 & $0.979-1.020$ & 0.936 \\
\hline \multicolumn{6}{|l|}{ Mean $\pm S D$} \\
\hline Tumor location, n (\%) & & & 0.972 & $0.622-1.518$ & 0.899 \\
\hline$\leq 5 \mathrm{~cm}$ to anus & $11(26.8 \%)$ & $59(11.7 \%)$ & & & \\
\hline$>5 \mathrm{~cm}$ to anus & $30(73.2 \%)$ & $445(88.3 \%)$ & & & \\
\hline Stoma, n (\%) & & & 2.533 & $1.438-4.463$ & $0.001^{*}$ \\
\hline No & $22(19.3)$ & $115(35.4)$ & & & \\
\hline Yes & $92(80.7)$ & $210(64.6)$ & & & \\
\hline Surgery, n (\%) & & & 0.795 & $0.493-1.281$ & 0.345 \\
\hline Open & $24(58.5)$ & $417(82.7)$ & & & \\
\hline Laparoscopic & $17(41.5)$ & $87(17.3)$ & & & \\
\hline
\end{tabular}


Table 5

Clinical characteristics of the patients who received non-operative treatment

\begin{tabular}{|c|c|c|}
\hline & $\begin{array}{l}\text { Stenosis } \\
\mathrm{N}=1\end{array}$ & $\begin{array}{l}\text { No stenosis } \\
\mathrm{N}=32\end{array}$ \\
\hline \multicolumn{3}{|l|}{ Age $(y)$} \\
\hline Mean $\pm S D$ & 36 & $56.8 \pm 9.6$ \\
\hline \multicolumn{3}{|l|}{ Sex, n (\%) } \\
\hline Male & $1(100)$ & $25(78.1)$ \\
\hline Female & $0(0)$ & 7 (21.9) \\
\hline \multicolumn{3}{|c|}{$\begin{array}{l}\text { Tumor location } \\
\text { (distance to anus, cm) }\end{array}$} \\
\hline Mean $\pm S D$ & 1 & $3.6 \pm 1.5$ \\
\hline \multicolumn{3}{|l|}{ Smoking, n (\%) } \\
\hline Yes & $0(0)$ & $1(3.1)$ \\
\hline No & $1(100)$ & $31(96.9)$ \\
\hline \multicolumn{3}{|c|}{ Concurrent Chemo, n (\%) } \\
\hline No & 0 & 0 \\
\hline Capecitabine & $1(100)$ & $5(15.6)$ \\
\hline Irinotecan+cape & 0 & $25(78.1)$ \\
\hline Others & 0 & $2(6.3)$ \\
\hline \multicolumn{3}{|c|}{ Follow-up times (m) } \\
\hline Mean $\pm S D$ & 26.6 & $25.2 \pm 21.6$ \\
\hline
\end{tabular}

\section{Fewer stenosis appear in patients received non-operative management}

Non-operative management for lower rectal cancer patients with a cCR after neoadjuvant chemoradiotherapy is a valuable alternative for rectal resection in recent years. In our center, watch and wait strategy used in lower rectal cancer patients from 2015. To explain if stenosis will appear in patients who received non-operative management when achieve CCR after neoadjuvant chemoradiotherapy. Thirty-three patients received non-operative management and had colonoscopy follow-up were retrospectively analyzed. The mean coloscopy follow time was more than 2 years. Of the 33 patients 
received non-operative treatment, only one experienced stenosis. The rate of stenosis was much lower in non-operative patients than in ARR patients

\section{Discussion}

Radiotherapy is an important management among multidisciplinary treatment in rectal cancer, reducing local recurrence and increasing organ preservation. Radiotherapy plays more important roles in immunotherapy era. However, radiotherapy is always challenged by its adverse effect, such as proctitis and stenosis. In most of the views, rectal stenosis results from pelvic radiotherapy. However, patients without receiving radiotherapy also suffer stenosis. Adverse effects cannot be simply attribute to radiotherapy when patients receiving multidisciplinary treatments. We were interested to answer the question that which factors are associated with rectal stenosis and which is the most important factor. We studied three groups of patients to explain this issue: patients receiving neoadjuvant chemoradiotherapy followed by ARR, patients receiving ARR without neoadjuvant or adjuvant radiothrapy and patients receiving non-operative management after achieving CCR. From our data, both radiotherapy and preventive ileostomy were implicated in rectal stenosis and were independent risk factors.

It has been reported that the incidence of rectal stenosis ranges from 2 to $30 \%$ [11-14]. The incidence of stenosis was $26.0 \%$ in patients with preoperative radiotherapy and $7.5 \%$ in patients without radiotherapy in our center. The incidence of stenosis is in accordance with the reported range of 2 to $30 \%$. The absence of a precise definition may account for this wide range. It is high in the preoperative radiotherapy group in our center. There are some reasons to explain this finding. First, we recorded all the patients who had difficulty in passing a 12-mm colonoscopy, including asymptomatic patients. Second, radiotherapy contributes to stenosis; the high rate of stenosis was in the preoperative group. Preoperative radiotherapy is widely used in our center for patients with locally advanced disease. Finally, patients who received preoperative radiotherapy were more likely to have a lower tumor location and undergo preventive ileostomy; after that, the occurrence of stenosis was more frequent.

In most of the views, pelvic irradiation induces rectal stenosis [6]. According to our univariate analysis, males, smoking and ileostomy were significant risk factors for stenosis in the preoperative chemoradiotherapy group. Multivariate analysis confirmed the significance of ileostomy, as $30.5 \%$ of patients with ileostomy experienced stenoses, while the incidence was only $16.1 \%$ in the non-stomy group $(P<0.001)$. In non-radiotherapy patients, stoma was also the only independent factor in multivariate analysis $(\mathrm{OR}=2.533, P=0.001)$. Preventive ileostomy was the only independent factor associated with stenosis in both preoperative radiotherapy and non-radiotherapy patients in our study. Most patients with resectable tumors would undergo surgery either after neoadjuvant radio- or chemotherapy or as the first step of the treatment. The common and dangerous complication is anastomotic leakage, particularly in tumors located not far from the anal verge. Due to the fistula's high rate, most authors recommend performing a loop ileostomy for protecting anastomoses $[3,16]$. The stomy will be closed several months later. Some patients will lose the chance of stoma closure for several reasons, such as distant metastasis 
and stenosis. Interestingly, the rate of stenosis was very low in patients with non-operative management, it indicated that radiotherapy is not the main factor to rectal stenosis.

We found that most of the stenoses occurred above the anastomosis instead of at the anastomosis. MRI images showed that the thickness of the bilateral obturator interrus increased significantly after chemoradiotherapy compared to pretreatment [17]. Rectal stenosis may be caused by radiation-induced fibrosis of the pelvic wall soft tissue. Muscle fibrosis may restrict the movement of the rectum, and this lack of motion led to stenosis. On the other way, pelvic nerve damage induced by surgery or radiotherapy will limit the motion of the rectum too [18]. It was revealed that males and smoking are stenosis risk factors in our univariate analysis in preoperative patients. It agrees with the results of Kim MJ and Bannura GC, i.e., that a history of heavy smoking was significantly associated with anastomotic complications, such as leakage and stricture $[19,20]$. Smoking exerts a negative effect on tissue oxygen supply through several mechanisms. Ischemia at the anastomosis site can cause anastomotic leakage or stricture by impeding the healing process.

The identification of risk factors for anastomotic complications can help decrease their frequency. Potential risk factors associated with rectal stenosis are preoperative radiotherapy and preventive ileostomy. Preoperative radiotherapy is confirmed as a standard treatment for locally advanced rectal cancer because it reduces local recurrence compared with postoperative radiotherapy $[11,21]$. Thus, it cannot be omitted. Preventive ileostomy reduced the occurrence of leakage. Stools were not excreted through the rectum in patients with an ileostomy; pelvic fibrosis can easily form because the rectum and pelvic muscles cannot move. The longer the preventive ileostomy remains, the more easily the fibrosis and stenosis will occur. In our study, the restoration of preventive stomy for more than six months will increase the occurrence of stenosis. Early closure of the protective ileostomy and anal functional training may be essential to reduce stenosis after surgery. Patients do not receive restoration during adjuvant chemotherapy result to late closure of preventive ileostomy. According to the trial of IDEA [22], some patients with low risks need fewer chemotherapy cycles and can receive early closure of stomy. Recently, the total neoadjuvant therapy (TNT) has been showing increased pCR and outcomes [23, 24]. In this new mode of treatment, patients do not need postoperative chemotherapy or they need less. Thus, preventive ileostomy can be closed early. Some anti-fibrotic medicines have been evaluated by pre-clinical studies. Several clinical trials have shown some effects on radiation-induced fibrosis or other fibrotic diseases, such as idiopathic pulmonary fibrosis [25-27]. However, these have not been widely used as a standard treatment at the clinic for radiation fibrosis. More effective and tolerable anti-fibrotic durgs should be studied for use at the clinic to reduce stenosis.

There are limitations in this study. First, this is a retrospective study, there can be a recall bias, and the symptoms associated with stenosis did not record. A further limitation is that stenosis could not be graded according to retrospective colonoscopy reports.

\section{Conclusion}


From our data, both radiotherapy and preventive ileostomy were implicated in rectal stenosis and were the independent risk factors, and radiotherapy is not the main factor. Restoration of the preventive stomy after more than six months will increase the occurrence of stenosis. Early closure of the protective ileostomy and anal functional training may be essential to reduce stenosis after surgery. More effective and tolerable anti-fibrotic drugs should be studied and used at the clinic to reduce stenosis.

\section{Abbreviations}

ARR: anterior rectal resection; ORs: odds ratios; CRC: Colorectal cancer; TME: total mesorectal excision; APR: abdominoperineal resection; IMRT: intensity-modulated radiation therapy; CTV: clinical tumor volume; cCR: clinical complete response; BMI: body mass index; TRG: tumor regression grade; $\mathrm{PCR}$ : pathologic complete response; SD: standard deviation; ROC: the receiving operator characteristic; TNT: total neoadjuvant therapy

\section{Declarations}

\section{Ethical approval and consent to participate}

The work was in accordance with The Code of Ethics of the World Medical Association (Declaration of Helsinsi) and was approved by the hospital's Medical Ethics Committee.

\section{Consent for publication}

Not applicable.

\section{Availability of supporting data}

The data used for analyzed during the current study are available from the corresponding author on reasonable request.

\section{Competing interests}

The authors declare that they have no competing interests.

\section{Funding}

This study was supported by the grants from the National Natural Science Foundation of China (Grant No. 81773357) and Wu Jieping Medical foundation (HYHX2021010).

\section{Authors' contributions}

Hui Zhang wrote the manuscript and performed procudures; Shanshan Li and Xin Jin collected the clinical data of each patient and wrote the manuscript; Xian Wu, Zhiyuan Zhang and Lijun Shen collected the clinical data; Juefeng Wan, Yan Wang, Yaqi Wang, Wang Yang and Menglong Zhou performed the 
statistical analysis; Jing Zhang, Tao Lv and Yun Deng drafted conception; Fan Xia and Zhen Zhang drafted conception and revised the manuscript. All authors read and approved the final manuscript.

\section{Acknowledgments}

We thank LetPub (www.letpub.com) for its linguistic assistance during the preparation of this manuscript.

\section{References}

1. Siegel RL, Miller KD, Jemal A. Cancer statistics, 2020. CA Cancer J Clin 2020; 70: 7-30.

2. AB B, AP V, MM A-H et al. Rectal Cancer, Version 2.2018, NCCN Clinical Practice Guidelines in Oncology. Journal of the National Comprehensive Cancer Network: JNCCN 2018; 16: 874-901.

3. Chude GG, Rayate NV, Patris V et al. Defunctioning loop ileostomy with low anterior resection for distal rectal cancer: should we make an ileostomy as a routine procedure? A prospective randomized study. Hepatogastroenterology 2008; 55: 1562-1567.

4. Luchtefeld M, Milsom J, Senagore A et al. Colorectal anastomotic stenosis. Results of a survey of the ASCRS membership. Diseases of the colon and rectum 1989; 32: 733-736.

5. Qin QY, Ma TH, Deng YH et al. Impact of Preoperative Radiotherapy on Anastomotic Leakage and Stenosis After Rectal Cancer Resection: Post Hoc Analysis of a Randomized Controlled Trial. Diseases of the colon and rectum 2016; 59: 934-942.

6. Zhu H, Bai B, Shan L et al. Preoperative radiotherapy for patients with rectal cancer: a risk factor for non-reversal of ileostomy caused by stenosis or stiffness proximal to colorectal anastomosis. Oncotarget 2017; 8: 100746-100753.

7. RJ M, MC G, I EN et al. Elective clinical target volumes for conformal therapy in anorectal cancer: a radiation therapy oncology group consensus panel contouring atlas. International journal of radiation oncology, biology, physics 2009; 74: 824-830.

8. Cai G, Zhu J, Palmer J et al. CAPIRIIIMRT: a phase II study of concurrent capecitabine and irinotecan with intensity-modulated radiation therapy for the treatment of recurrent rectal cancer. Radiation oncology (London, England) 2015; 10: 57.

9. Zhu J, Li X, Shen Y et al. Genotype-driven phase I study of weekly irinotecan in combination with capecitabine-based neoadjuvant chemoradiation for locally advanced rectal cancer. Radiotherapy and oncology : journal of the European Society for Therapeutic Radiology and Oncology 2018; 129 : 143-148.

10. Zhu J, Liu A, Sun X et al. UGT1A1Multicenter, Randomized, Phase III Trial of Neoadjuvant Chemoradiation With Capecitabine and Irinotecan Guided by Status in Patients With Locally Advanced Rectal Cancer. Journal of clinical oncology : official journal of the American Society of Clinical Oncology 2020; JCO2001932. 
11. Sauer $\mathrm{R}$, Becker $\mathrm{H}$, Hohenberger $\mathrm{W}$ et al. Preoperative versus postoperative chemoradiotherapy for rectal cancer. N Engl J Med 2004; 351: 1731-1740.

12. Kim CW, Kim JH, Yu CS et al. Complications after sphincter-saving resection in rectal cancer patients according to whether chemoradiotherapy is performed before or after surgery. Int J Radiat Oncol Biol Phys 2010; 78: 156-163.

13. Polese $L$, Vecchiato $M$, Frigo AC et al. Risk factors for colorectal anastomotic stenoses and their impact on quality of life: what are the lessons to learn? Colorectal Dis 2012; 14: e124-128.

14. Schlegel R, Dehni N, Parc R et al. Results of reoperations in colorectal anastomotic strictures. Diseases of the colon and rectum 2001; 44: 1464-1468.

15. Lee SY, Kim CH, Kim YJ, Kim HR. Anastomotic stricture after ultralow anterior resection or intersphincteric resection for very low-lying rectal cancer. Surg Endosc 2018; 32: 660-666.

16. Huser N, Michalski CW, Erkan M et al. Systematic review and meta-analysis of the role of defunctioning stoma in low rectal cancer surgery. Ann Surg 2008; 248: 52-60.

17. Chi P, Chen Z, Gao Y et al. [Diagnosis and treatment of pelvic wall and bowel fibrosis with bowel obstruction induced by neoadjuvant chemoradiotherapy for rectal carcinoma]. Zhonghua wei chang wai ke za zhi $=$ Chinese journal of gastrointestinal surgery 2015; 18: 1092-1097.

18. Da Silva GM, Berho M, Wexner SD et al. Histologic analysis of the irradiated anal sphincter. Dis Colon Rectum 2003; 46: 1492-1497.

19. MJ K, R S, HK O et al. The impact of heavy smoking on anastomotic leakage and stricture after low anterior resection in rectal cancer patients. World journal of surgery 2011; 35: 2806-2810.

20. Bannura G, Cumsille M, Barrera A et al. Predictive factors of stenosis after stapled colorectal anastomosis: prospective analysis of 179 consecutive patients. World journal of surgery 2004; 28 : 921-925.

21. Sauer R, Liersch T, Merkel S et al. Preoperative versus postoperative chemoradiotherapy for locally advanced rectal cancer: results of the German CAO/ARO/AIO-94 randomized phase III trial after a median follow-up of 11 years. J Clin Oncol 2012; 30: 1926-1933.

22. André T, Vernerey D, Mineur L et al. Three Versus 6 Months of Oxaliplatin-Based Adjuvant Chemotherapy for Patients With Stage III Colon Cancer: Disease-Free Survival Results From a Randomized, Open-Label, International Duration Evaluation of Adjuvant (IDEA) France, Phase III Trial. Journal of clinical oncology: official journal of the American Society of Clinical Oncology 2018; 36: 1469-1477.

23. Fokas $E$, Allgäuer $M$, Polat B et al. Randomized Phase II Trial of Chemoradiotherapy Plus Induction or Consolidation Chemotherapy as Total Neoadjuvant Therapy for Locally Advanced Rectal Cancer: CAO/ARO/AIO-12. Journal of clinical oncology : official journal of the American Society of Clinical Oncology 2019; 37: 3212-3222.

24. Cercek A, Roxburgh C, Strombom P et al. Adoption of Total Neoadjuvant Therapy for Locally Advanced Rectal Cancer. JAMA oncology 2018; 4: e180071. 
25. Hille A, Christiansen $\mathrm{H}$, Pradier $\mathrm{O}$ et al. Effect of pentoxifylline and tocopherol on radiation proctitis/enteritis. Strahlenther Onkol 2005; 181: 606-614.

26. Gothard L, Cornes P, Brooker S et al. Phase II study of vitamin E and pentoxifylline in patients with late side effects of pelvic radiotherapy. Radiother Oncol 2005; 75: 334-341.

27. Bourgier C, Auperin A, Rivera S et al. Pravastatin Reverses Established Radiation-Induced Cutaneous and Subcutaneous Fibrosis in Patients With Head and Neck Cancer: Results of the Biology-Driven Phase 2 Clinical Trial Pravacur. International journal of radiation oncology, biology, physics 2019; 104: 365-373.

\section{Figures}

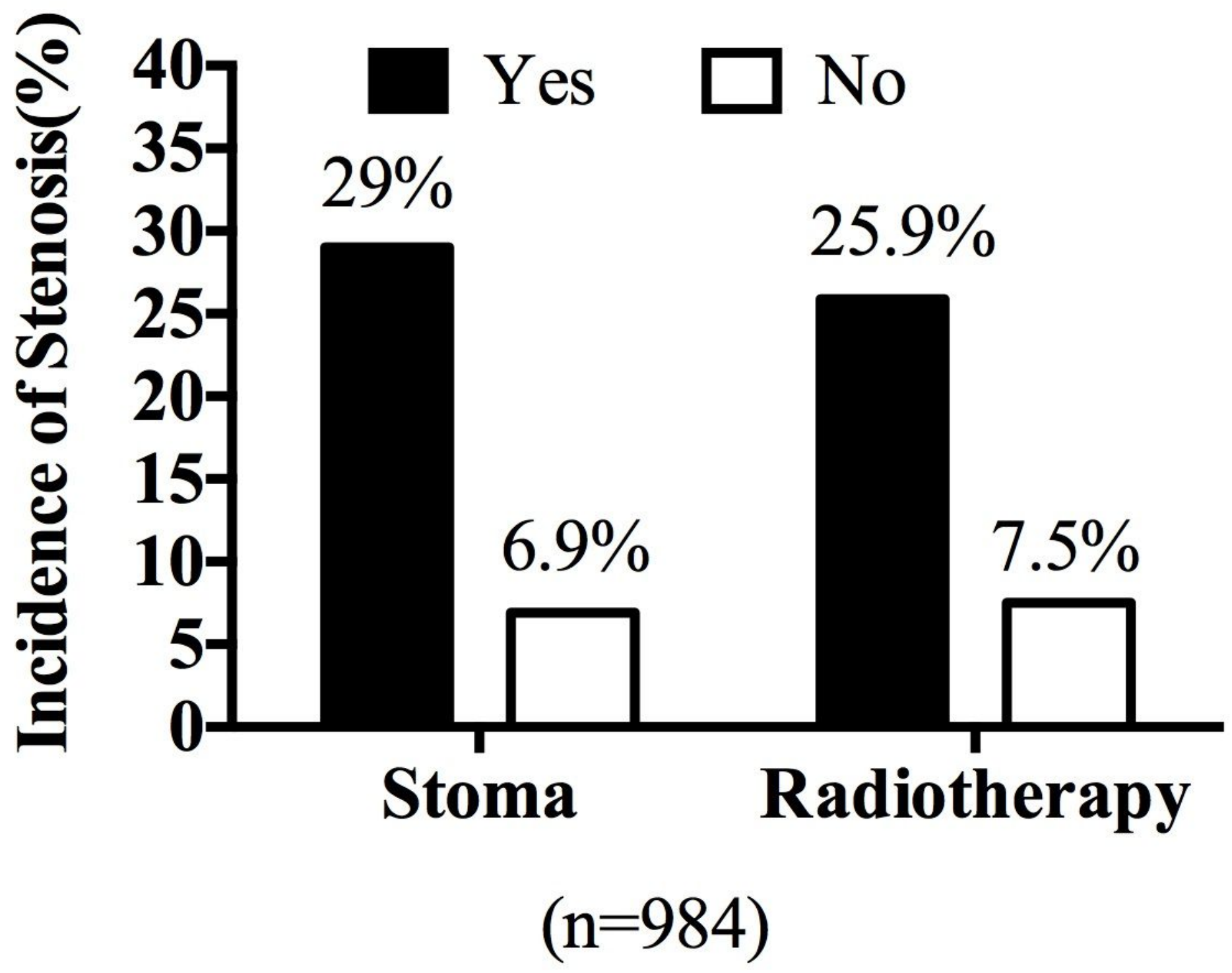

Figure 1 
The incidence of stenosis in patients received preventive ileostoma or preoperative radiotherapy. 114 diagnosed with stenoses in patients with stoma, and 279 did not, the rate of stenoses was $114 /(114+279)=29 \%$. And the rate of stenoses was $6.9 \%$ in no stoma patients. The rate of stenoses was $26.0 \%(114 /(114+324))$ and $7.5 \%(41 /(41+504))$ in radiotherapy and non-radiotherapy groups, respectively.

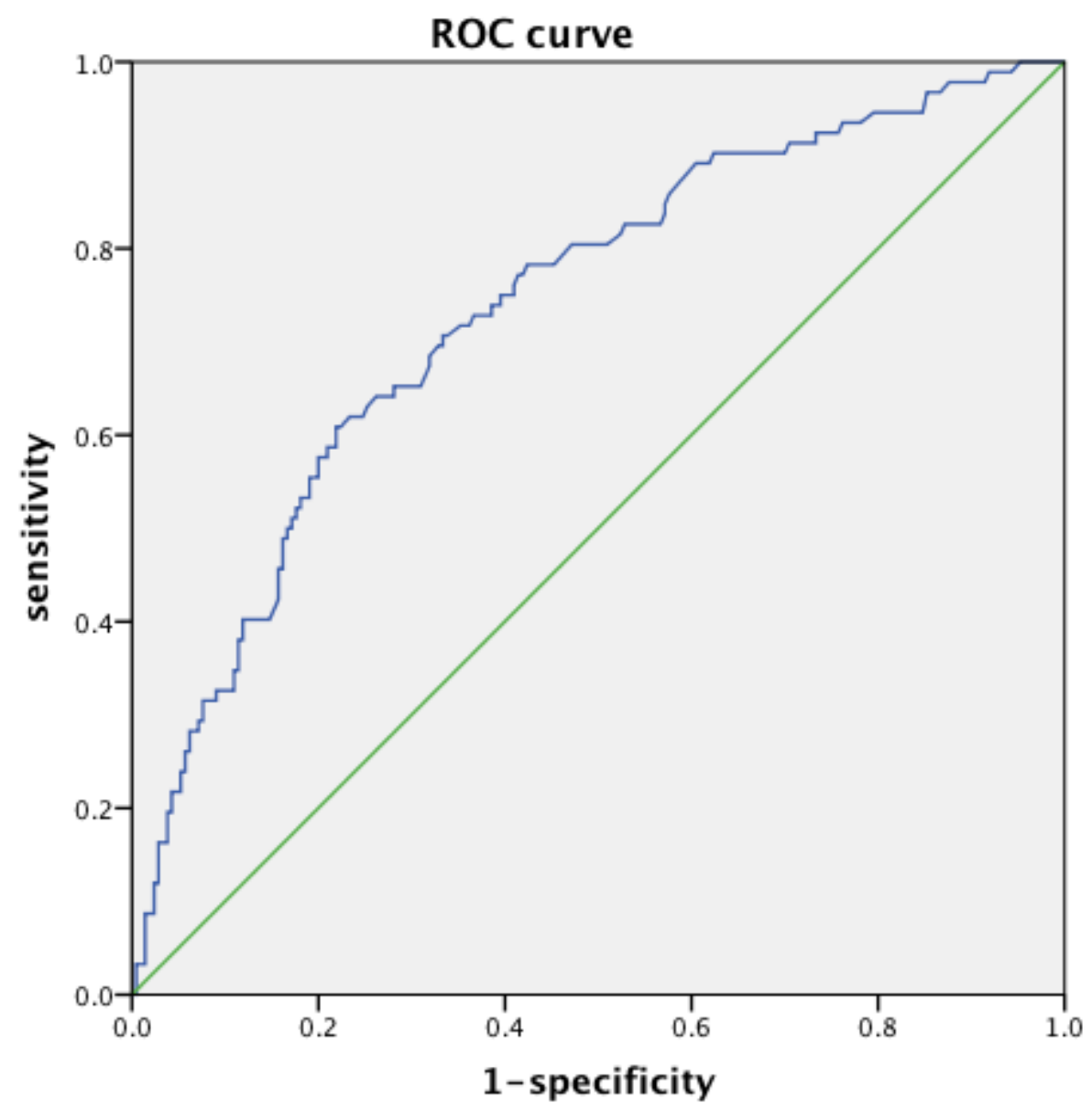

\section{Figure 2}

The ROC curve for the cut-off point of the interval between ileostomy and restoration to predicting ractal stenosis.

\section{Supplementary Files}

This is a list of supplementary files associated with this preprint. Click to download.

- Supplementarymaterial.docx 